\title{
El laboratorio en el diagnóstico multidisciplinar del desarrollo sexual anómalo o diferente (DSD)
}

\author{
I) Fisiología, clasificación, abordaje y metodología \\ II) Marcadores bioquímicos y genéticos diagnósticos en los 46,XX
}

https://doi.org/10.1515/almed-2020-0119

Received December 14, 2020; accepted February 24, 2021; published online May 24, 2021

\section{Resumen}

Objetivos: El desarrollo de las características sexuales femeninas o masculinas acontece durante la vida fetal, determinándose el sexo genético, el gonadal y el sexo genital interno y externo (femenino o masculino). Cualquier discordancia en las etapas de diferenciación ocasiona un desarrollo sexual anómalo o diferente (DSD) que se clasifica según la composición de los cromosomas sexuales del cariotipo.

Contenido: En este capítulo se abordan la fisiología de la determinación y el desarrollo de las características sexuales femeninas o masculinas durante la vida fetal, la clasificación general de los DSD y su estudio diagnóstico clínico, bioquímico y genético que debe ser multidisciplinar. Los estudios bioquímicos deben incluir, además de las determinaciones bioquímicas generales, análisis de hormonas esteroideas y peptídicas, en condiciones basales o en pruebas funcionales de estimulación. El estudio genético debe comenzar con la determinación del cariotipo al que seguirá un estudio molecular en los cariotipos 46,XX ó $46, X Y$, orientado a la caracterización de un gen candidato. Además, se expondrán de manera específica los marcadores bioquímicos y genéticos en los DSD 46,XX, que incluyen el

\footnotetext{
*Autor para correspondencia: Dr. María Luisa Granada, MD, PhD, Department of Clinical Biochemistry, Hospital Germans Trias i Pujol, Autonomous University of Barcelona, Crta. Canyet s/n, 08916, Badalona, España, E-mail: granadaybern@gmail.com Laura Audí, Growth and Development Research Group, Vall d'Hebron Research Institute (VHIR), Center for Biomedical Research on Rare Diseases (CIBERER), Instituto de Salud Carlos III, Barcelona, Catalonia, España
}

desarrollo gonadal anómalo (disgenesias, ovotestes y testes), el exceso de andrógenos de origen fetal (el más frecuente), fetoplacentario o materno y las anomalías del desarrollo de los genitales internos.

Perspectivas: El diagnóstico de un DSD requiere la contribución de un equipo multidisciplinar coordinado por un clínico y que incluya los servicios de bioquímica y genética clínica y molecular, un servicio de radiología e imagen y un servicio de anatomía patológica.

Palabras clave: desarrollo sexual anómalo o diferente (DSD); diagnóstico bioquímico; diagnóstico genético; DSD $46, \mathrm{XX}$.

\section{Fisiología, clasificación, abordaje y metodología}

\section{1) Fisiología de la diferenciación sexual y clasificación de sus variaciones}

La determinación y el desarrollo de las características sexuales femeninas y masculinas durante la vida fetal constituyen procesos biológicos complejos que incluyen la expresión de cascadas de genes cuyas proteínas ejercen funciones altamente específicas, tanto en su localización como en su cronología [1-3]. El sexo genético se establece en el momento de la fertilización al fusionarse un espermatozoide (con un cromosoma sexual $\mathrm{X}$ o Y), con un ovocito (cromosoma $\mathrm{X}$ ), dando lugar a una célula diploide 46,XX o 46,XY que determina el sexo genético. Durante las primeras semanas del embrión, el desarrollo de las estructuras gonadales y genitales es común a ambos sexos. El desarrollo del seno urogenital y del primordio adrenogonadal acontece hacia la $4^{\mathrm{a}}$ semana. El desarrollo de la gónada primitiva bipotencial requiere la expresión de una cascada de genes (entre ellos bien caracterizados en humanos: EMX2, CBX2, NR5A1, GATA4, WT1) [1, 3]. 
A partir de la $6^{\text {a }}$ semana, la presencia de un cromosoma Y y la expresión de su gen $S R Y$ activa una cascada de genes que regulan el desarrollo de la gónada indiferenciada hacia un testículo [4] e inhibe la expresión de los genes que feminizan la gónada hacia un ovario $[1,3,5,6]$. El desarrollo gonadal depende de complejas interacciones entre genes antagónicos que regulan procesos que finalmente determinan la formación de un testículo o de un ovario [7] (Figura 1).

En su etapa inicial indiferenciada, ambos sexos comparten un par de conductos genitales (conductos de Müller y conductos de Wolff) así como la morfología de los genitales externos (tubérculo genital y repliegues genitales) [8]. El desarrollo de genitales internos y externos en el sentido masculino depende de que el testículo secrete una serie de hormonas en cantidades adecuadas y siguiendo una cronología determinada. Los genitales internos masculinos requieren la secreción y acción de testosterona (T) que induce la diferenciación de los conductos de Wolff en epidídimo y conductos deferentes y de la hormona antiMülleriana $(\mathrm{AMH})$ que actúa a través de su receptor (AMHR2) y provoca la regresión de los conductos de Müller [8]. El desarrollo de la próstata y de los genitales externos requiere el metabolismo local de la $\mathrm{T}$ que se transforma en dihidrotestosterona (DHT) por acción de la enzima 5-alfareductasa tipo 2 [9] (Figura 1).

En ausencia de AMH y de concentraciones elevadas de andrógenos (T y DHT) se desarrollan los genitales internos y externos femeninos (Figura 1). Aunque el modelo murino de ausencia de receptor de estrógenos (ER) puede sugerir que los estrógenos podrían actuar a través del receptor ER feminizando el tubérculo genital [10], la morfología de los genitales externos de una RN con resistencia completa al estradiol (E2) no ha sido descrita hasta la fecha.

La presencia de alteraciones en alguna de las etapas puede modificar el desarrollo sexual normal produciéndose "anomalías o diferencias en el desarrollo sexual” (DSD) (“disorders or differences of sex development") [11],

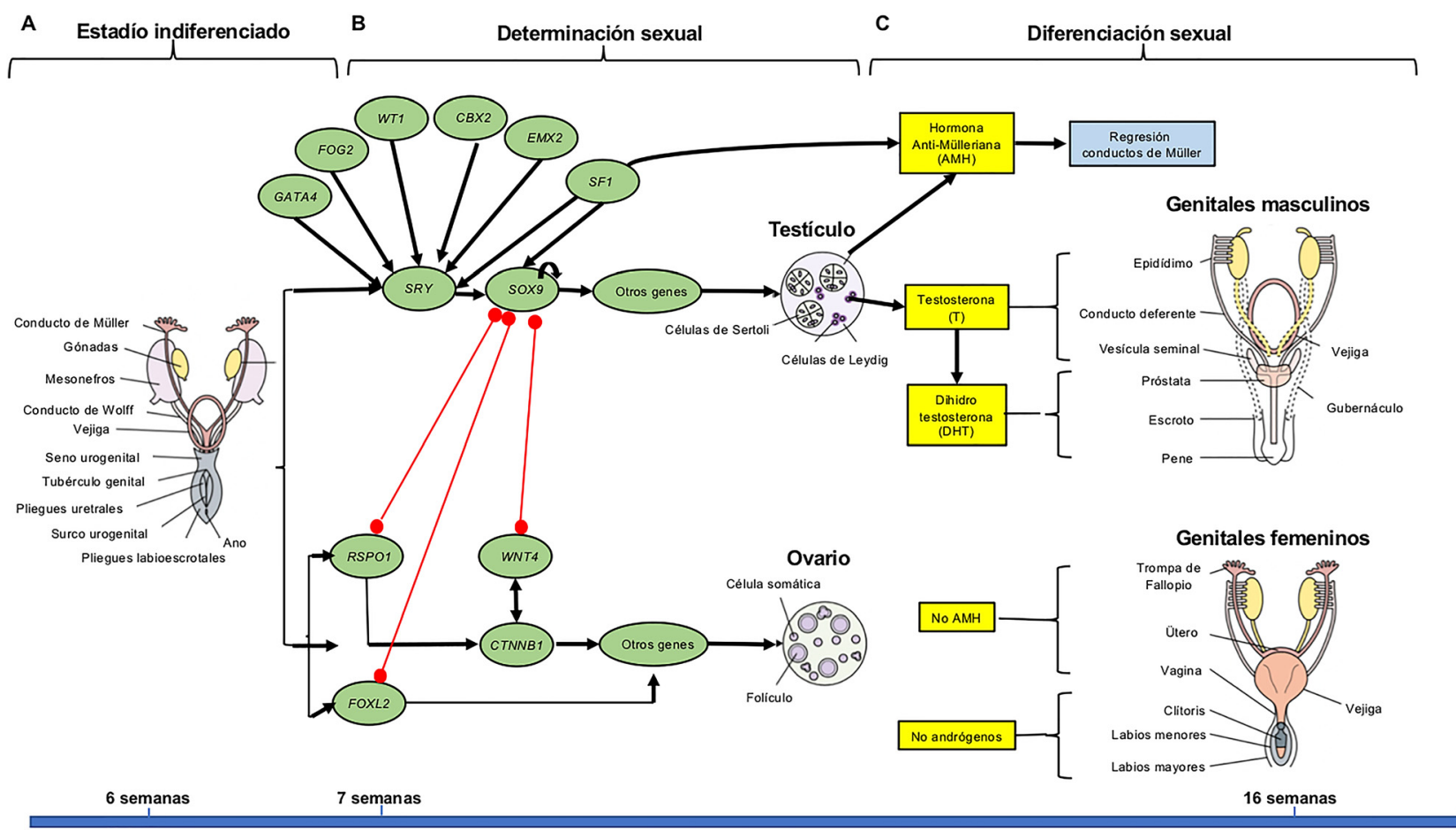

Semanas de gestación

Figura 1: Desarrollo sexual humano durante la vida fetal.

(A) Estadío indiferenciado: Las gónadas bipotenciales están desarrolladas a las 5 semanas, así como dos pares de conductos internos (conductos de Múller y de Wolff), los genitales externos constan del tubérculo genital, los pliegues uretrales que flanquean el surco urogenital, y los pliegues labioescrotales. Este estadío termina hacia la 6a semana. (B) Determinación sexual: Comienza a desarrollarse entre las semanas 6 $6^{\underline{a}}$ y $7^{\underline{a}}$ cuando las células somáticas y los gonocitos de las gónadas bipotenciales se diferencían en células testiculares u ováricas, dependiendo de la presencia y activación o represión de vías de señalización. Las flechas indican la activación de genes mientras que las líneas rojas indican represión de la expresión génica. (C) Diferenciación sexual: La diferenciación de genitales internos y externos depende de la presencia o ausencia de hormonas testiculares (hormona anti-Mülleriana [AMH], testosterona [T] y dihidrotestosterona [DHT]). Modificado de cita: León et al. [16]. 
Tabla 1: Clasificación del desarrollo sexual anómalo o diferente (DSD) según los cromosomas sexuales presentes en el cariotipo [11].

\section{1) DSD por cromosomas sexuales}

(1) 47,XXY: síndrome de Klinefelter y variantes

(2) 45,X0 y mosaicos 45,X0/46,XX (síndrome de Turner y variantes)

(3) $45, X 0 / 46, X Y$ mosaico (disgenesia gonadal mixta)

(4) $46, X X / 46, X Y$ mosaico (DSD ovotesticular)

(5) $47, \mathrm{XYY}$

\section{2) DSD con cariotipo $46, \mathrm{XX}$}

1. Desarrollo gonadal anómalo

2. Desarrollo genital anómalo por exceso de andrógenos

\author{
a. Disgenesia gonadal parcial (DGP) o completa (DGC) \\ b. DSD ovotesticular \\ c. DSD testicular
}

Producción fetal:

a. Déficit de 21-hidroxilasa

b. Déficit de $3 \beta$-hidroxiesteroide deshidrogenasa tipo 2

c. Déficit de 11- $\beta$-hidroxilasa

d. Resistencia a glucocorticoides

e. Resistencia a estrógenos

Producción feto-placentaria:

a. Déficit de P450 oxido-reductasa

b. Déficit placentario y fetal de aromatasa

c. Tumores fetales o placentarios productores de andrógenos

Origen materno:

a. Agentes terapéuticos o contaminantes medioambientales

b. HSC materna

c. Tumores maternos virilizantes (luteomas, tumor de Krukenberg)

3. Desarrollo anómalo de los genitales internos

a. Síndrome pie-mano-genital

b. MURCS (Müllerian aplasia, Renal aplasia, Cervico-thoracic Somite abnormalities)

c. MRKH (Mayer-Rokitansky-Küster-Hauser) syndrome, tipos I y II

\section{3) DSD con cariotipo $46, X Y$}

1. Desarrollo gonadal anómalo

2. Desarrollo genital anómalo por anomalías en la síntesis o en la acción de los andrógenos

3. Desarrollo genital anómalo por anomalías en la síntesis o en la acción de la hormona anti-Mülleriana (AMH)

\section{Síndromes malformativos complejos}
a. Disgenesia gonadal parcial (DGP) o completa (DGC)
b. DSD ovotesticular
c. DSD ovárico

\section{omalías en la síntesis de andrógenos:}

a. Insensibilidad a la LH (aplasia/hipoplasia de células de Leydig)

b. Déficit de 7-dehidrocolesterol reductasa (síndrome de Smith-Lemli-Opitz)

c. Déficit de proteína StAR (hiperplasia suprarrenal congénita lipoidea)

d. Déficit de colesterol desmolasa

e. Déficit de 33-hidroxi-esteroide deshidrogenasa tipo 2

f. Déficit de $17 \alpha$-hidroxilasa/17-20 desmolasa

g. Déficit de $\mathbf{P} 450$ oxidoreductasa

h. Déficit de citocromo B5

i. Déficit en la esteroidogénesis de la vía trasera

j. Déficit de 17ß-hidroxi-esteroide deshidrogenasa tipo 3

k. Déficit de $5 \alpha$-reductasa tipo 2

l. Hipospadias y/o criptorquidia aislados

Anomalías en la acción de los andrógenos:

a. Insensibilidad completa o parcial a los andrógenos

b. Agentes terapéuticos o contaminantes medioambientales

Persistencia de los conductos de Müller:

a. Déficit de hormona anti-Mülleriana

b. Resistencia a la hormona anti-Mülleriana

a. Síndromes malformativos con desarrollo genital anómalo (anomalías cloacales, síndrome de Aarskog, síndrome de Robinow, etc.)

b. Crecimiento intrauterino retardado, precoz y severo 
condiciones congénitas en las que el desarrollo de los sexos cromosómico, gonadal y/o genital es atípico o distinto al más frecuente.

Las causas de DSD se clasifican según el Consenso de Chicago [11] en función del cariotipo. Existen tres grupos principales: - 1) los DSD cromosómicos, cuando la composición de los cromosomas sexuales es distinta de los pares XX o $\mathrm{XY}$; - 2) los DSD 46,XX con cariotipo femenino, - 3) los DSD 46,XY con cariotipo masculino (Tabla 1). Cada grupo se subdivide en distintos tipos (Tabla 1). En los Grupos 2 y 3, los genes implicados son muy numerosos y aumentan de año en año.

En el Grupo 1, los DSD cromosómicos quedan definidos por el número o la estructura de los cromosomas sexuales (Tabla 1). Los más frecuentes son el 47,XXY (síndrome de Klinefelter), el 45,X0 (síndrome de Turner) y sus variantes, incluido el mosaico 45,X/46,XY (o disgenesia gonadal mixta), el mosaico 46,XX/46,XY (verdadera quimera sexual cromosómica o DSD ovotesticular) y el 47,XYY.

En el Grupo 2, 46,XX, pueden existir (Tabla 1): - 1) Anomalías en el desarrollo gonadal (disgenesia gonadal completa [DGC], parcial [DGP], quimera ovotesticular [DSD ovotesticular] o desarrollo testicular [DSD testicular]); - 2) Desarrollo genital anómalo por exceso de andrógenos (de producción fetal, feto-placentaria o de origen materno) que virilizan los genitales externos; - 3) Desarrollo anómalo de los genitales internos.

En el Grupo 3, con cariotipo 46,XY pueden existir (Tabla 1): - 1) Anomalías en el desarrollo gonadal (DGC, DGP, desarrollo ovotesticular [DSD ovotesticular] o desarrollo ovárico [DSD ovárico]); - 2) Anomalías en la síntesis o en la acción de los andrógenos; - 3) Anomalías en la síntesis o en la acción de la AMH; - 4) Síndromes malformativos complejos que afectan el desarrollo genitourinario y digestivo y el crecimiento intrauterino retardado, precoz y severo que se acompaña de hipospadias.

Excepto para los DSD cromosómicos del Grupo 1 (sobre todo el síndrome de Klinefelter con cariotipo $47, \mathrm{XXY}$ ) y los niños con cariotipo 46,XY del Grupo 3 que nacen con hipospadias, la frecuencia poblacional para los DSD de los Grupos 2 y 3 es tan baja que entran dentro de la categoría de las llamadas "enfermedades raras" (ER) (frecuencia poblacional inferior a 1/2.000).

\section{2) Equipos multidisciplinares para el diagnóstico de los DSD}

El DSD puede manifestarse en el recién nacido $(\mathrm{RN})$ o poco después, por la presencia de genitales externos ambiguos, por discordancia entre el cariotipo prenatal y el desarrollo genital, cuando existe una historia familiar de DSD, cuando se presenta una insuficiencia suprarrenal aguda o cuando se detecta la presencia de una gónada en una hernia inguinal. Posteriormente, durante el desarrollo puberal, por discordancias entre el desarrollo gonadal y el genital. Además, algunos adultos no diagnosticados pueden consultar por infertilidad o por otros problemas de salud como una hipertensión arterial y la investigación de las posibles etiologías conducir a la detección de algún DSD.

Diagnosticar la causa de un DSD puede ser más o menos complejo, dependerá, en parte, de los conocimientos y la habilidad de cada profesional involucrado así como de la funcionalidad del equipo multidisciplinar de especialistas [11, 12]. En todos los protocolos se insiste en que el diagnóstico médico de un DSD requiere la contribución de un equipo de especialistas que colaboren con un clínico coordinador [12, 13], y que incluye un servicio de bioquímica (bioquímica general y marcadores $\mathrm{u}$ hormonas específicas), un servicio de genética clínica y molecular (cariotipo inicial y la interpretación del resultado de otras exploraciones orientarán el desarrollo de otros análisis), un servicio de radiología e imagen (una ecografía pélvica para detectar las estructuras genitales internas y la presencia de gónadas intraabdominales) y un servicio de anatomía patológica (cuando se requiere el análisis de la estructura de las gónadas).

Se han elaborado y propuesto muchos algoritmos diagnósticos [13-16] que han ido evolucionando en función de las tecnologías asequibles, principalmente en los campos de la imagen y la bioquímica, pero sobre todo del diagnóstico molecular [17-21].

\section{3) Exploraciones bioquímicas y genéticas en el diagnóstico de los DSD}

\section{a) Exploraciones bioquímicas basales}

Las pruebas bioquímicas y, en especial, las magnitudes hormonales juegan un papel muy importante tanto en el diagnóstico inicial de los DSD como en el control evolutivo y la monitorización del tratamiento. Podemos diferenciar dos grandes grupos de hormonas: las hormonas esteroideas y las peptídicas.

Las hormonas esteroideas (Figura 2) se sintetizan a partir del colesterol en el córtex adrenal, las gónadas y la placenta aunque se metabolizan en numerosos tejidos periféricos. Su medición en sangre y en orina ha ido evolucionando hasta las técnicas actuales de inmunoensayo y de espectrometría de masas. Disponemos de inmunoensayos comerciales para los esteroides más solicitados en la práctica clínica; sin embargo, para algunos parámetros de 
interés en el diagnóstico de los DSD como la corticosterona, la desoxicorticosterona, la 17-OH-pregnenolona y la DHT se deberá recurrir a cromatografía tándem líquida-espectrometría de masas (LC-MS/MS) [22, 23]. Las sociedades científicas internacionales recomiendan la utilización de métodos basados en la espectrometría de masas (LC-MS/MS y GC-MS/MS) para la medida de esteroides sexuales y sus precursores, en el diagnóstico de los DSD, en especial, en los neonatos [24]. Esta metodología permite determinar en una misma muestra perfiles de esteroides, incluyendo metabolitos para los que no se dispone de inmunoensayos específicos [25]. Los esteroides se pueden medir en diferentes matrices: suero, sangre, saliva, orina [26]. Es muy importante que el laboratorio trabaje según estándares de calidad, que participe en programas externos de calidad y que disponga de intervalos de referencia específicos de edad y sexo [24]. Los esteroides adrenales tienen un marcado ritmo de secreción circadiano por lo que se aconseja realizar las determinaciones en muestras obtenidas a primera hora de la mañana (8-9 a.m.).

La medición de hormonas proteicas se realiza fundamentalmente por inmunoensayos no competitivos que tienen gran sensibilidad, pero cuya especificidad no siempre es conocida. La falta de métodos de referencia y las diferencias

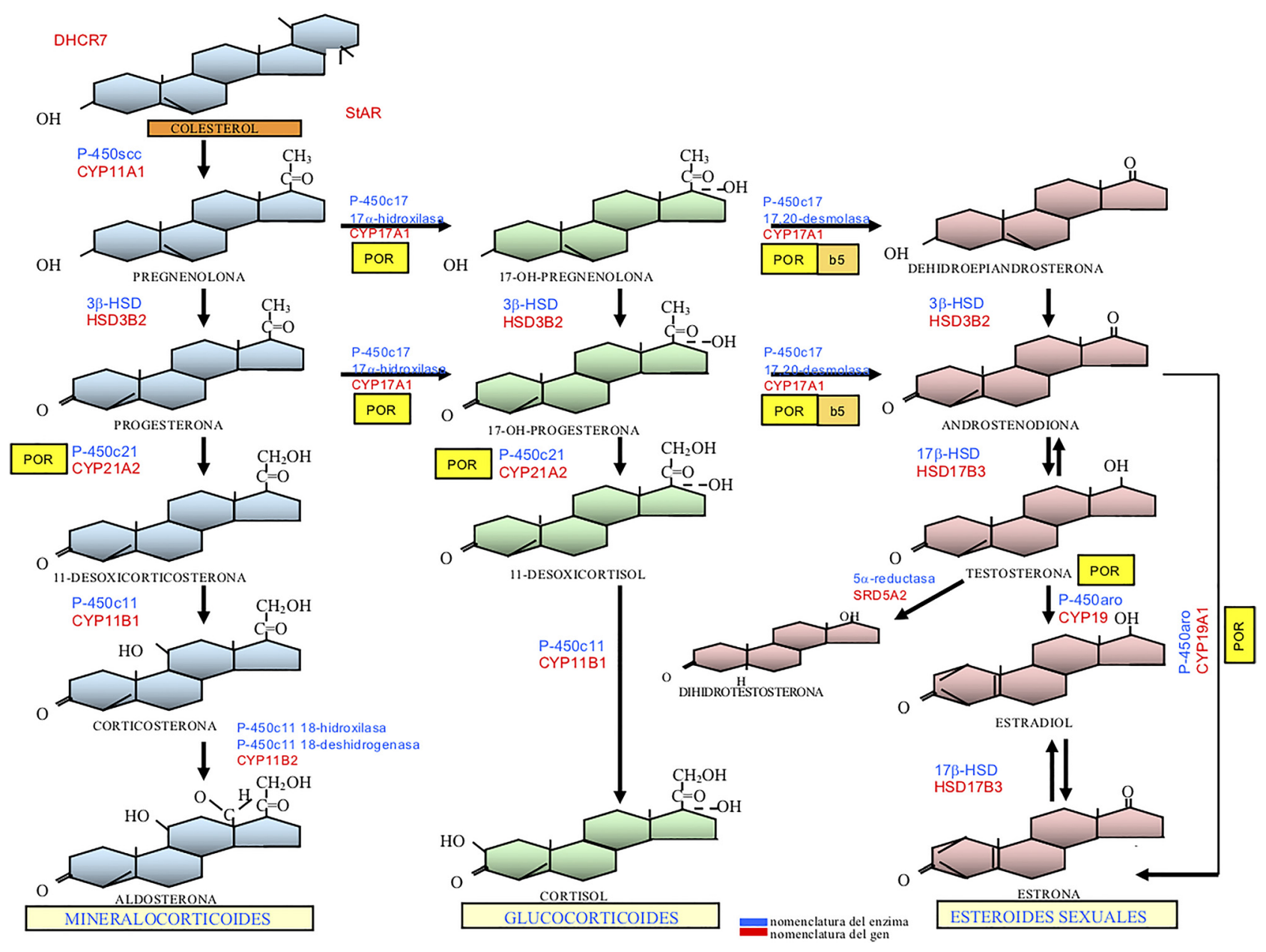

Figura 2: Esteroidogénesis suprarrenal y gonadal.

Desde el colesterol, la biosíntesis progresa en la glándula suprarrenal hasta el cortisol (vía de los glucocorticoides) y hasta la aldosterona (vía de los mineralocorticoides). En las gónadas los precursores progresan hacia los esteroides sexuales: testosterona (T) como principal andrógeno y estradiol como principal estrógeno. T es transformada periféricamente en dihidrotestosterona (DHT) como andrógeno más potente. En azul: abreviación de los enzimas; en rojo: abreviación de los genes que codifican cada enzima; cuadro amarillo: coenzima POR (P450-oxidoreductasa); cuadro beis: citocromo b5. DHCR7 = gen DHCR7 (7alfa-dehidrocolesterol reductasa) StAR = steroid acute regulatory protein (gen StAR) P-450scc $=$ P-450 side-chain cleavage $=$ colesterol-desmolasa (gen CYP11A1) $3 \beta-H S D=3 \beta$-hidroxiesteroidedeshidrogenasa tipo 2 (gen HSD3B2) P-450c17 = 17 $\alpha$-hidroxilasa/17,20-desmolasa o liasa (gen CYP17A1) P-450c21 = 21-hidroxilasa (gen CYP21A2) P-450c11 = 11 $\beta$-hidroxilasa tipo 1 (gen CYP11B1) P-450c11 18-hidroxilasa (corticosterona metil-oxidasa tipo 1 [CMO-I]) y P-450c11 18-deshidrogenasa (corticosterona metil-oxidasa tipo II [CMO-II]) (gen CYP11B2) 17ß-HSD = 17-hidroxiesteroide deshidrogenasa tipo 3 ó 17-cetorreductasa (gen HSD17B3) 5 $\alpha$-reductasa tipo 2 (gen SRD5A2) P-450aro = aromatasa (gen CYP19A1). 
en la estandarización se traducen en una falta de comparabilidad entre ensayos haciendo indispensable disponer de valores de referencia específicos para los diferentes grupos de edad y sexo para cada ensayo [27, 28]. Las hormonas peptídicas relacionadas con el desarrollo sexual presentan un marcado dimorfismo sexual. En los primeros años de vida en el sexo masculino las concentraciones de LH son más elevadas que las de FSH y el cociente LH/FSH es claramente más elevado [29]. Las concentraciones de AMH son 100 veces más elevadas en el varón que en la mujer y las de INHB 10 veces superiores. Recientemente se ha publicado una guía para la determinación de hormonas peptídicas en el estudio de los DSD [30].

\section{b) Pruebas funcionales}

En algunos casos la medición de hormonas basales no es suficientemente informativa y es necesario realizar pruebas de estimulación para poner en evidencia déficits de secreción.

Se realizan fundamentalmente tres tipos de pruebas funcionales:

b-1) Prueba de estimulación con Corticotropina (ACTH) [31] $\mathrm{Su}$ objetivo es el estudio de la esteroidogénesis adrenal (Figura 2). Se administra por vía endovenosa ACTH sintético (1-24) (Cosyntropin o Synacthen $®$ ) a dosis de $0,25 \mathrm{mg}$ (en bebés se puede reducir a 0,125 mg). Se determinan diferentes hormonas y precursores basalmente y a los 60 minutos del estímulo para valorar déficits enzimáticos adrenales [32].

\section{b-2) Prueba de estimulación con gonadotropina coriónica (HCG) [33]}

La HCG estimula la producción de andrógenos testiculares al unirse al receptor de LH-HCG de las células de Leydig. Existen diferentes protocolos de estimulación. La medida de andrógenos y sus precursores se realiza antes y a las 48-72 horas de la última inyección. En el diagnóstico de DSD es importante medir T, sus precursores y su metabolito DHT para diagnosticar déficits enzimáticos en la esteroidogénesis testicular y periférica (Figura 2).

\section{b-3) Prueba de estimulación con LHRH o sus análogos}

Se puede realizar administrando el factor hipotalámico LHRH, liberador de LH y FSH, (Prueba de Luforan ${ }^{\circledR} 100 \mu \mathrm{g}$ i.v y en niños de 25 a $50 \mu$ g) o más recientemente, utilizando análogos del LHRH (acetato de leuprorelina [Procrin ${ }^{\circledR}$ ], acetato de buserelina). Una respuesta de LH superior a 5
UI/L se considera indicativo de activación central del eje hipotálamo-hipófiso-gonadal (HHG) [34-36].

\section{c) Exploraciones genéticas}

\section{c-1) Citogenética y cariotipo}

El cariotipo es la base para la clasificación de un DSD en alguno de los tres grupos diagnósticos que depende de los cromosomas sexuales presentes (Tabla 1). La técnica clásica es de citogenética, aunque recientemente se están utilizando técnicas de hibridación de alta resolución [21].

Además de anomalías en los cromosomas sexuales, algunos DSD pueden ser portadores de variaciones en el número de copias (VNC) (deleciones, duplicaciones, translocaciones), tanto en autosomas como en cromosomas sexuales; esto es especialmente importante cuando el fenotipo incluye otras anomalías adicionales al DSD [37-40]. Las VNC se detectan mediante técnicas de hibridación de alta resolución (array-CGH: array-complementary genome hybridisation) y podrán ser detectadas al analizar el cariotipo mediante técnicas de array-CGH.

\section{c-2) Análisis de genes}

Las causas monogénicas de DSD más frecuentes se fueron describiendo a lo largo del último tercio del siglo XX cuando se fueron clonando genes que codifican proteínas cuya alteración era conocida por los fenotipos clínico y bioquímico. Esto es especialmente cierto para los déficits enzimáticos de la esteroidogénesis adrenal y gonadal (Figura 2), tanto en los DSD 46,XX como en los 46,XY, y en el síndrome de insensibilidad completa a los andrógenos. En cambio, los genes implicados en la determinación y el desarrollo de las gónadas masculina y femenina van siendo progresivamente detectados a partir de estudios familiares, modelos animales $\mathrm{y}$ estudios funcionales in vitro $[7,41]$. Un buen número de genes relacionados con el desarrollo de un DSD codifican factores de transcripción reguladores de otros genes (por ejemplo: AR, DAX1, DMRT1, FOXL2, NR5A1, SOX3, SOX9 y $S R Y)$ y, habiéndose demostrado la existencia de mutaciones en regiones no-codificantes del genoma, se puede anticipar que la exploración del genoma no-codificante permitirá la progresiva comprensión de la causa de algunos DSD que carecen de diagnóstico molecular [42].

El análisis estructural de un gen candidato individual se realiza mediante secuenciación automática de Sanger que comporta la amplificación mediante PCR de las regiones codificantes y flanqueantes y, eventualmente, de la región promotora. Sin embargo, la introducción de técnicas secuenciación de alto rendimiento permite actualmente el análisis del exoma completo (regiones codificantes) o 
seleccionar el análisis de un panel de genes candidatos. Además, la amplia expresividad en los fenotipos de los DSD podría explicarse en algunos casos por una afectación oligogénica en la que la interacción de varios genes relacionados puede ser responsable de un fenotipo único para cada individuo [43]. La mejora progresiva de la calidad de estas técnicas y de su precio, las han hecho accesibles a la mayor parte de laboratorios de diagnóstico molecular, de modo que el análisis de un gen individual o de una de sus regiones quedará progresivamente limitado al diagnóstico de un nuevo paciente familiar de un caso bien caracterizado [21, 44-49].

\section{Marcadores bioquímicos y genéticos en los DSD 46,XX}

La Tabla 2 expone la lista de causas monogénicas de DSD en el Grupo con cariotipo 46,XX. La lista se va alargando de año en año, sobre todo entre las causas de desarrollo gonadal disgenético.

\section{1) Desarrollo gonadal anómalo}

Las anomalías en el desarrollo gonadal con cariotipo 46, XX comprenden las disgenesias gonadales parciales y completas (DGP y DGC), las gónadas ovotesticulares (DSD ovotesticular) y el desarrollo de testículo (DSD testicular) (Tabla 1).

Las más frecuentes son las DGP y DGC. Ninguna de las dos da lugar a ambigüedad genital, siendo el fenotipo al nacer femenino. Suelen manifestarse clínicamente en la pubertad, cuando ésta se retarda y/o no se desarrolla. Los marcadores bioquímicos muestran aumento de la LH y FSH, AMH indetectable y estradiol (E2) a concentraciones prepuberales. Otros precursores como la androstendiona, la 17-hidroxiprogesterona (17OH-P) y la T también tendrán concentraciones prepuberales, mientras que la dehidroepiandrosterona (DHEA) y su sulfato habrán aumentado durante la adrenarquia normal. Una forma clínica más leve y relativamente frecuente es la menopausia precoz o fallo ovárico prematuro, siendo sus marcadores bioquímicos el aumento precoz de LH y FSH, una disminución de la AMH

Tabla 2: Diagnósticos clínicos y genes involucrados en el desarrollo sexual anómalo o diferente (DSD) de causa monogénica.

\section{DSD con cariotipo $46, \mathrm{XX}$}

\begin{tabular}{|c|c|c|}
\hline $\begin{array}{l}\text { Diagnóstico clínico } \\
\text { (from clinico untilö adicional) }\end{array}$ & Gen (locus) & OMIM (herencia) (fenotipo adicional) \\
\hline \multicolumn{3}{|c|}{ 1. DSD $46, X X$ por anomalías en el desarrollo gonadal: disgenesia gonadal, DSD ovotesticular, DSD testicular } \\
\hline Disgenesia gonadal & $B M P 15(\mathrm{Xp} 11.22)$ & $300510 / 300247$ (D) \\
\hline Disgenesia gonadal & ESR2 (14q23.2-q23.3) & $618187(\mathrm{AD})$ \\
\hline DSD testicular & FGF9 (13q12.11) & 600921 (AD:dup) (Un solo caso descrito) \\
\hline Disgenesia gonadal & FOXL2 (3q22.3) & $\begin{array}{l}608996 \text { (AD) (Blefarofimosis, epicanto inverso y ptosis, } \\
\text { tipos I y II) }\end{array}$ \\
\hline \multirow[t]{2}{*}{ Disgenesia gonadal } & MYRF (11q12.2) & 608329 (AD) \\
\hline & & 618280 (AD) (Síndrome cardíaco urogenital) \\
\hline DSD testicular & NR2F2 $(15 q 26.2$ & $\begin{array}{l}615779 \text { (AD) (cardiopatía congénita, hernia dia- } \\
\text { fragmática, síndrome blefarofimosis-ptosis-epicanto } \\
\text { inverso) }\end{array}$ \\
\hline 1) Disgenesia gonadal & 1) $N R 5 A 1(9 q 33.3)$ & 612964 (AD) \\
\hline 2) DSD ovotesticular & 2) $N R 5 A 1(9 q 33.3)$ (p.Arg92Trp) & $617480(A D)$ \\
\hline 3) DSD testicular & 3) NR5A1 (p.Arg92Trp) & \\
\hline Disgenesia gonadal & NUP107 (12q15) & $\begin{array}{l}607617 \text { (AR) (descrito en familia consanguínea; otros } \\
\text { fenotipos con síndrome nefrótico) }\end{array}$ \\
\hline DSD ovotesticular & RSPO1 (1p34.3) & $\begin{array}{l}610644 \text { (AR) (Hiperqueratosis palmoplantar, carci- } \\
\text { noma cutáneo de células escamosas) }\end{array}$ \\
\hline 1) DSD ovotesticular & $\operatorname{sox3}(\mathrm{Xq} 27.1)$ & 313430 (XL:dup) \\
\hline \multicolumn{3}{|l|}{ 2) DSD testicular } \\
\hline Disgenesia gonadal & SOX8 (16p13.3) & 605923 (Insuficiencia ovárica primaria) \\
\hline 1) DSD ovotesticular & $\operatorname{SOX9}(17 \mathrm{q} 24.3)$ & 278850 (AD:dup) \\
\hline \multicolumn{3}{|l|}{ 2) DSD testicular } \\
\hline 1) DSD ovotesticular & $\operatorname{SOX10}(22 \mathrm{q} 13.1)$ & 609136 (AD:dup) (síndromes de Waardenberg y de \\
\hline \multirow[t]{2}{*}{ 2) DSD testicular } & & Hirschsprung, neuropatía periférica) \\
\hline & $S R Y(Y p 11.2)$ & $400045(\mathrm{~T})$ \\
\hline
\end{tabular}


Tabla 2: (continued)

\section{DSD con cariotipo $46, \mathrm{XX}$}

Diagnóstico clínico

(from clinico untilö adicional)

1. DSD 46,XX por anomalías en el desarrollo gonadal: disgenesia gonadal, DSD ovotesticular, DSD testicular

1) DSD ovotesticular

2) DSD testicular

1) DSD ovotesticular

2) DSD testicular

1) DSD ovotesticular

2) DSD testicular
Gen (locus)

OMIM (herencia) (fenotipo adicional)

2. DSD 46,XX con desarrollo gonadal normal pero genital anómalo por exceso de andrógenos fetales o fetoplacentarios

\begin{tabular}{|c|c|c|}
\hline HSC por déficit de 21-hidroxilasa & CYP21A2 (6p21.33) & 201910 (AR) (Déficit suprarrenal) \\
\hline $\begin{array}{l}\text { HSC por deficit de } 3 \alpha \text {-hidroxi-esteroide deshi- } \\
\text { drogenasa tipo } 2\end{array}$ & HSD3B2 (1p12) & 201810 (AR) (Déficit suprarrenal y gonadal) \\
\hline HSC por déficit de $11 \beta$-hidroxilasa & CYP11B1 (8q24.3) & 202010 (AR) (Déficit suprarrenal) \\
\hline Insensibilidad a los glucocorticoides & $G R \alpha(N R 3 C 1) 5 q 31.3$ & 615962 (AD) (Hipertensión) \\
\hline Insensibilidad a los estrógenos & ESR1 (6q25.1-q25.2) & $\begin{array}{l}615363 \text { (AR) (Hipercrecimiento, osteoporosis, ovario } \\
\text { poliquístico) (Un solo caso descrito) }\end{array}$ \\
\hline Déficit de P450-oxidoreductasa & POR (7q11.23) & $\begin{array}{l}201750 \text { (AR) } \\
\text { (déficit de } 17 \alpha \text {-hidroxilasa, 21-hidroxilasa y aromatasa } \\
\text { variables) } \\
\text { (síndrome de Antley-Bixler syndrome, } \pm \\
\text { craniosinostosis) }\end{array}$ \\
\hline Déficit de aromatasa & CYP19A1 (15q21.2) & 613546 (AR) (virilización materna y fetal) \\
\hline
\end{tabular}

3. DSD 46,XX con desarrollo gonadal normal pero anómalo de los conductos Müllerianos

Síndrome Pie-Mano-Genital

Síndrome MURCS (Müllerian Aplasia, Renal aplasia, Cervico-thoracic Somite abnormalities) Síndrome MRKH (Mayer-Rokitansky-KüsterHauser), tipos I y II

Aplasia Mülleriana e hiperandrogenismo

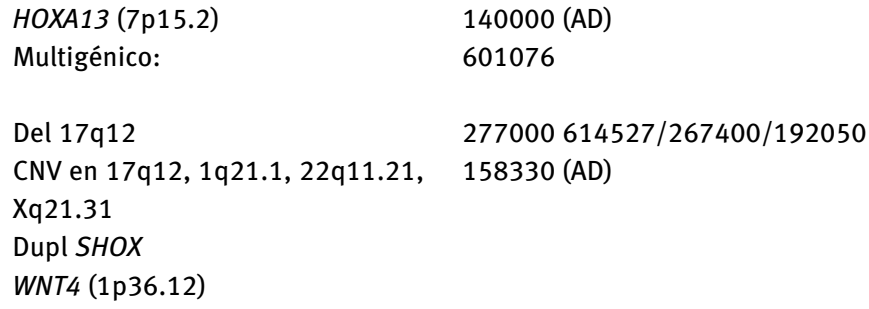

DSD, desarrollo sexual anómalo o diferente; DGP, disgenesia gonadal parcial; DGC, disgenesia gonadal completa; HSC, hiperplasia suprarrenal congénita; D, dominante; AD, autosómico dominante; AR, autosómico recesivo; XL, ligado al X; T, translocación; Dup, duplicación; Del, deleción; ¿?, desconocido; CNV, variación en número de copias.

(buen marcador de la reserva ovárica de folículos), la disminución de E2 y progesterona (P). Se van describiendo causas monogénicas (Tabla 2), entre ellas mutaciones inactivadoras en los genes BMP15, ESR2, FOXL2, MYRF, NR5A1, NUP107 y SOX8. En la mayoría de casos el efecto es dominante (excepto NUP107) y en algunos casos se asocian otras características fenotípicas (Tabla 2).

El desarrollo ovotesticular o testicular presenta ambigüedad genital (incluso genitales externos completamente masculinos) desde el nacimiento, ya que ha existido exposición a concentraciones elevadas de $\mathrm{T}$ durante el desarrollo fetal. Los marcadores bioquímicos en el $\mathrm{RN}$ o lactante serán similares a los hallados en el sexo masculino $(46, \mathrm{XY})$; durante la infancia la capacidad de secreción de $\mathrm{T}$ por las gónadas se podrá evaluar mediante el test de HCG. En la pubertad se produce un aumento de $\mathrm{T}$, que no alcanza niveles masculinos normales por lo que se acompaña de un aumento de LH y FSH. La mayoría de causas monogénicas pueden dar lugar tanto a DSD ovotesticular como testicular (Tabla 2). La primera conocida fue la translocación de un fragmento del cromosoma Y que contenga el gen $S R Y$ sobre un autosoma. Algunos se asocian a fenotipos complejos como las mutaciones en NR2F2, RSPO1, SOX1O, WNT4 y recientemente WT1; en el caso del gen NR5A1, sólo la mutación p.Arg92Trp 
provoca el desarrollo ovotesticular o testicular; también se describen duplicaciones en los genes FGF9, SOX3 y SOX9.

\section{2) Desarrollo genital anómalo por exceso de andrógenos}

Cuando el desarrollo gonadal es ovárico y el de los genitales internos es femenino, el exceso de andrógenos durante el desarrollo fetal produce la virilización de los genitales externos. El origen de los andrógenos puede ser el propio feto, la unidad fetoplacentaria o la madre (Tabla 1).

\section{a) Aumento de andrógenos de origen fetal}

En la mayoría de los $\mathrm{RN}$ con cariotipo 46,XX que presentan virilización de los genitales externos, ésta es debida a una hiperplasia suprarrenal congénita (HSC). La causa más frecuente es el déficit de 21-hidroxilasa [32, 50] (gen CYP21A2), que en su forma "simple virilizante" presenta un déficit de cortisol, aumento de ACTH, acúmulo del precursor 17OH-P, de androstendiona y de T (Figura 2). En las formas más graves, o "perdedoras de sal”, se asocia un déficit de aldosterona con hiponatremia, hiperkaliemia y aumento de la actividad de la renina plasmática (ARP). El diagnóstico bioquímico se realiza ante el hallazgo de concentraciones elevadas de $17 \mathrm{OH}-\mathrm{P}$ (basal o post ACTH $>300 \mathrm{nmol} / \mathrm{L}$ [ $>10.000 \mathrm{ng} / \mathrm{dL}]$ ), androstendiona y T. También es necesario medir glucosa y electrolitos en sangre y la ARP [51]. Es importante tener valores de referencia estratificados por edad gestacional ya que en el RN prematuro las concentraciones de $17 \mathrm{OH}-\mathrm{P}$ son mucho más elevadas, siendo causa de diagnósticos falsamente positivos [52]. La determinación del 21-deoxicortisol en suero, producido por la $11 \beta$-hidroxilación de la $17 \mathrm{OH}-\mathrm{P}$, puede ser útil para minimizar los diagnósticos falsos positivos ya que se eleva en el déficit de 21-hidroxilasa pero no en otros déficits adrenales ni en los RN prematuros, aunque es una determinación que no está disponible en muchos laboratorios clínicos [53].

\section{VÍAS DE BIOSÍNTESIS DE ANDRÓGENOS}

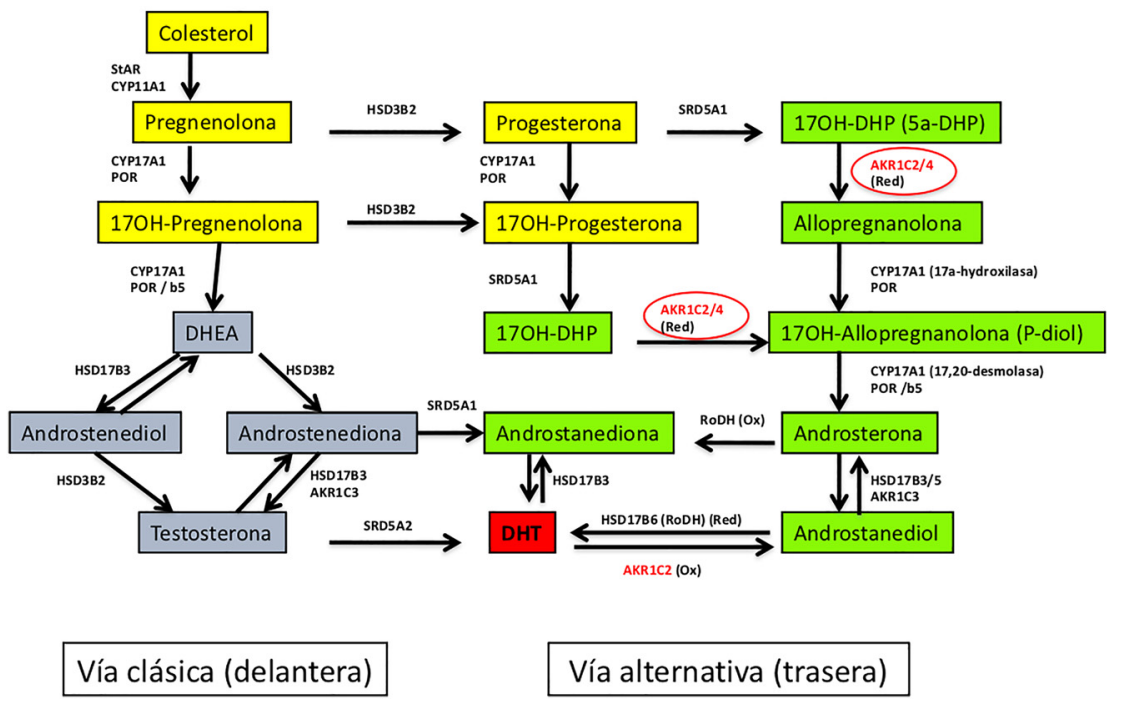

Figura 3: Vías de biosíntesis de andrógenos: vía clásica (o delantera) y vía alternativa (o trasera).

La vía clásica progresa desde el colesterol hasta la testosterona (T), pasando por la pregnenolona (intervienen la proteína StAR [gen STAR] y el enzima colesterol desmolasa [gen CYP11A1]), la 17-OH-pregnenolona (enzima 17 $\alpha$-hidroxilasa [gen CYP17A1] y coenzima P450-oxidoreductasa [gen POR]), la dehidroepiandrosterona (DHEA) (enzima 17,20-desmolasa [gen CYP17A1], coenzima P450-oxidoreductasa [gen POR] y citocromo b5 [gen CYB5A]), la androstenediona o el androstenediol (enzimas 3 $\beta$-hidroxiesteroide deshidrogenasa tipo 2 [gen HSD3B2], 17beta-

hidroxiesteroide deshidrogenasa tipo 3 [gen HSD17B3] y aldo-keto reductasa familia 1 miembro C3 [gen AKR1C3]). La T es transformada en dihidrotestosterona (DHT) mediante el enzima $5 \alpha$-reductasa tipo 2 (gen SRD5A2). También la DHT puede proceder de la transformación de androstenediona en androstanediona (enzima $5 \alpha$-reductasa tipo 1 [gen SRD5A1]) y de ésta en DHT (enzima 17 $\beta$-hidroxiesteroide deshidrogenasa tipo 3 [gen HSD17B3]). La vía alternativa llega a la síntesis de DHT sin pasar por la T. En ella, la progesterona (procede de la pregnenolona) y la $170 \mathrm{H}$-progesterona (procede de la progesterona) son transformadas en 170H-DHP (5 $\alpha$-dihidroxi-progesterona). Ésta llega a androsterona la cual pasa a androstanediona o androstanediol, las cuales pasan a DHT (intervienen varios enzimas previamente descritos así como aldo-keto reductasa familia 1 miembros C2 y C4 [genes AKR1C2 y AKR1C4], retinol-dehidrogenasa [gen RODH], 17ß-hidroxiesteroide deshidrogenasa tipo 5 y tipo 6 [genes HSD17B5 y HSD17B6]). (Ox) = oxidación; (Red) = reducción. 
La medida de esteroides urinarios mediante GC-MS/MS muestra la activación de la vía alternativa de la puerta trasera (alternative backdoor pathway) (Figura 3), con incremento del $5 \alpha$-pregnane-3 $\alpha, 17 \alpha$-diol-20-one (P-diol), pregnanetriol (P-triol), la $17 \mathrm{OH}$-pregnanolona y aumento del cociente androsterona/etiocolanolona [54].

Existen formas leves del déficit enzimático, llamadas "no clásicas" o tardías, que se manifiestan por una pubarquia precoz con discreta aceleración de la velocidad de crecimiento y de la maduración ósea, y aparición de vello pubiano. Bioquímicamente existe un discreto aumento de la 17OH-P basal con aumento o no de la androstendiona y de la T. La estimulación con ACTH demostrará el exceso de 170H-P (31-300 nmol/L; 1.000-10.000 ng/dL).

El diagnóstico molecular deberá confirmar la presencia de mutaciones en el gen CYP21A2, en homocigosis o en heterocigosis compuesta, cuyo efecto es la anulación completa o casi completa de la actividad enzimática en las formas severas, tanto con pérdida salina como en la virilizante simple. Existe una asociación entre el genotipo y el grado de virilización de las pacientes con formas clásicas del déficit enzimático [55]. En las formas "no clásicas" uno de los alelos debe ser portador de una mutación de efecto leve, que permita la síntesis de una cierta actividad enzimática. Las mutaciones en el gen CYP21A2 constituyen la anomalía molecular más frecuente en humanos, aunque su incidencia varía según las zonas geográficas y las estructuras sociales [32, 56] (Tabla 2).

Otras causas mucho menos frecuentes de HSC son el déficit de 33-hidroxiesteroide deshidrogenasa tipo 2 (3ßHSD2), el de 11 $\beta$-hidroxilasa y el déficit de citocromo $\mathrm{P}-450$ oxidorreductasa (POR) [57].

El enzima 3ßHSD2 (gen HSD3B2) cataliza dos reacciones secuenciales, transformando la pregnenolona en $\mathrm{P}$, la 17OH-pregnenolona en 17OH-P y la DHEA en androstendiona (Figura 2). Las pacientes con déficit grave presentan HSC por déficit en la síntesis de cortisol y de aldosterona. Existe un aumento de los esteroides $\Delta 5$ (pregnenolona, 170H-pregnenolona, DHEA) y de la relación de éstos con los esteroides $\Delta 4$ (P, 17OH-P y androstendiona). Sin embargo, las concentraciones de $170 \mathrm{OH}-\mathrm{P}$, pueden estar aumentadas por la conversión periférica de $\Delta 5-17 \mathrm{OH}$-pregnenolona por la enzima 33HSD tipo 1. El diagnóstico bioquímico se basa en la demostración de concentraciones elevadas de $170 \mathrm{H}$-pregnenolona ( $>150 \mathrm{nmol} / \mathrm{L}$ ) basal o tras estimulación con ACTH [58]. El diagnóstico molecular deberá confirmar la presencia en homocigosis o heterocigosis compuesta de mutaciones inactivadoras en el gen HSD3B2 (Tabla 2).

El enzima 11ß-hidroxilasa convierte el 11-desoxicortisol en cortisol y la 11-desoxicorticosterona en corticosterona
(Figura 2). Su déficit da lugar a una HSC con déficit de cortisol y de aldosterona que deriva a la síntesis de andrógenos (T) y se asocia a una virilización muy importante del feto femenino. El perfil hormonal se caracteriza por disminución de cortisol y de aldosterona, con aumento de ACTH pero ARP inhibida. El indicador diagnóstico más robusto es el aumento de 11-desoxicortisol y de 11-desoxicorticosterona, aunque estos parámetros no están disponibles en muchos laboratorios clínicos. Un 60 \% de las pacientes presenta hipertensión arterial por acúmulo de 11-desoxicorticosterona que tiene acción mineralocorticoide. El perfil de esteroides en orina muestra un patrón de metabolitos del cortisol reducido y un incremento de los metabolitos de la 11-desoxicorticosterona [59].

Existen formas leves del déficit enzimático [60]. El diagnóstico molecular deberá confirmar la presencia en homocigosis o heterocigosis compuesta de mutaciones en el gen CYP11B1 (Tabla 2).

La resistencia a los glucocorticoides es una causa muy infrecuente de virilización del feto femenino. A causa de una mutación en el gen del receptor de glucocorticoides (GR $\alpha$ o NR3C1) (Tabla 2) hay una hipersecreción de cortisol y de ACTH sin evidencia clínica de hipercortisolismo, pero con manifestaciones de exceso de andrógenos y de mineralocorticoides [61].

La resistencia a los estrógenos por mutaciones inactivadoras en el receptor alfa del E2 (gen ESR1) (Tabla 2) es una patología muy infrecuente, primero descrita en el sexo masculino; en el femenino, la morfología de los genitales externos al nacer no ha sido descrita pero desarrollan una virilización postnatal por desarrollo de un ovario poliquístico con aumento de androstendiona y $\mathrm{T}$, así como talla alta y osteoporosis [62].

\section{b) Aumento de andrógenos de origen fetoplacentario}

La P450-oxidorreductasa (POR), es una flavoproteína unida a la membrana del citocromo $C$ que juega un papel fundamental en la transferencia de electrones del NADPH a los enzimas microsomales P450 (CYP21, CYP17 y CYP19 o aromatasa). El déficit de POR se caracteriza por déficit parcial y muy variable de varias actividades enzimáticas (Figura 2): 17 $\alpha$-hidroxilasa y 17,20-desmolasa que puede $o$ no asociarse a déficit de 21-hidroxilasa y de aromatasa. Los pacientes pueden presentar un amplio espectro fenotípico y asociarse a malformaciones esqueléticas características (síndrome de Antley-Bixler). Puede ser causa de HSC y de ambigüedad genital [63]. Desde el punto de vista bioquímico pueden presentar concentraciones normales o bajas de cortisol, elevadas de $17 \mathrm{OH}-\mathrm{P}$, eventualmente de $\mathrm{T}$ y concentraciones anómalas de algunos de los esteroides 
(y sus metabolitos) de la "vía de la puerta trasera" (Figura 3). El perfil urinario de esteroides muestra el acúmulo de metabolitos de la pregnenolona y de la P. Las niñas afectadas nacen con genitales ambiguos por exceso de andrógenos intrauterinos debido al déficit de aromatasa (CYP19) y/o por la síntesis de DHT por la vía de la puerta trasera [64]. La madre puede haber manifestado signos de virilización durante el embarazo con concentraciones elevadas de T. La virilización de la niña no progresa y las concentraciones de andrógenos circulantes no son elevadas hasta que, al llegar la pubertad, puede manifestarse un déficit de síntesis ovárica de E2 y de nuevo un aumento de precursores de la vía trasera de la esteroidogénesis [65]. El diagnóstico molecular debe confirmar la presencia en homocigosis o heterocigosis compuesta de mutaciones inactivadoras en el gen POR (Tabla 2).

La enzima aromatasa (CYP19) cataliza el paso de $\mathrm{T}$ a E2 y de androstendiona a estrona (E1). En ausencia de actividad aromatasa la placenta no puede convertir el sulfato de DHEA, producido en grandes cantidades por la adrenal fetal, en estrógenos (E1, E2 y estriol) y se convierte en $\mathrm{T}$ lo que provoca la virilización del feto $46, \mathrm{XX}$ y de la madre [66]. Suele observarse un aumento de $\mathrm{T}$ y de gonadotropinas, en especial de la FSH. El diagnóstico molecular debe confirmar la presencia de mutaciones en homocigosis o heterocigosis compuesta en el gen CYP19A1 (Tabla 2).

Tumores fetales o placentarios productores de andrógenos: se ha descrito algún caso de tumor suprarrenal congénito responsable de la virilización de un feto 46,XX.

\section{c) Aumento de andrógenos de origen materno}

El exceso de andrógenos puede proceder de la madre por presencia de tumores virilizantes durante el embarazo (como el luteoma del embarazo o el tumor de Krukenberg), por tratamiento fármacológico o por contaminantes medioambientales con efecto androgénico o incluso ser debido a un mal control terapéutico durante el embarazo de una madre, afecta también de HSC (Tabla 1).

\section{3) Desarrollo anómalo de los genitales internos}

Existen malformaciones aisladas de los conductos genitales internos femeninos (útero, vagina y trompas de Falopio). Pueden ser debidas a un desarrollo incompleto o a la presencia de estructuras anómalas (Tabla 1). No son frecuentes $\mathrm{y}$, en algunos casos, presentan incidencia familiar, por lo que una posible causa genética puede ser invocada, aunque en escasas ocasiones llega a ser aclarada.
No existen marcadores bioquímicos específicos. Sólo la clínica de amenorrea, dismenorrea, infertilidad puede orientar el diagnóstico. Las malformaciones consisten en aplasia o hipoplasia de útero y trompas, útero bicorne o bipartito que pueden asociarse a malformaciones de otros sistemas o tejidos, como en el síndrome Pie-Mano-Genital (para el que se ha descrito un gen asociado como HOXA13), el síndrome MURCS (Müllerian aplasia, Renal aplasia, Cervico-thoracic Somite abnormalities, definido como multigénico hasta la actualidad) y el síndrome MRKH (Mayer-Rokitansky-Küster-Hauser) tipos I y II en el que se describen varias anomalías genéticas y finalmente las mutaciones inactivadoras en el gen WNT4 para las que hemos descrito un posible desarrollo ovotesticular o testicular y que también pueden presentar una aplasia de conductos Müllerianos (Tabla 2).

Contribución de los autores: Todos los autores aceptan su responsabilidad en relación al contenido del manuscrito y aprueban su presentación.

Conflicto de intereses: Los autores declaran no tener ningún conflicto de intereses.

Financiación de la investigación: No declarada. Consentimiento informado: No se aplica.

Aprobación ética: La Junta de Revisión Institucional local consideró que el estudio estaba exento de revisión.

\section{Referencias}

1. Eggers S, Sinclair A. Mammalian sex determination-insights from humans and mice. Chromosome Res 2012;20:215-38.

2. Munger SC, Natarajan A, Looger LL, Ohler U, Capel B. Fine time course expression analysis identifies cascades of activation and repression and maps a putative regulator of mammalian sex determination. PLoS Genet 2013;9:e1003630.

3. Eggers S, Ohnesorg T, Sinclair A. Genetic regulation of mammalian gonad development. Nat Rev Endocrinol 2014;10:673-83.

4. Berta P, Hawkins J, Sinclair A, Taylor A, Griffiths BL, Goodfellow PN, et al. Genetic evidence equating SRY and the testis-determining factor. Nature 1990;348:448-50.

5. Harley VR, Clarkson MJ, Argentaro A. The molecular action and regulation of the testis-determining factors, $S R Y$ (sex-determining region on the Y chromosome) and SOX9 [SRY-related high-mobility group (HMG) box 9]. Endocr Rev 2003;24:466-87.

6. Sekido R, Lovell-Badge R. Sex determination and SRY: down to a wink and a nudge? Trends Genet 2009;25:19-29.

7. Bashamboo A, Eozenou C, Rojo S, McElreavey K. Anomalies in human sex determination provide unique insights into the complex genetic interactions of early gonad development. Clin Genet 2017;91:143-56.

8. Zhao F, Yao HH. A tale of two tracts: history, current advances, and future directions of research on sexual differentiation of reproductive tracts. Biol Reprod 2019;101:602-16. 
9. Imperato-McGinley J, Zhu YS. Androgens and male physiology the syndrome of 5alpha-reductase-2 deficiency. Mol Cell Endocrinol 2002;198:51-9.

10. Yang JH, Menshenina J, Cunha GR, Place N, Baskin LS. Morphology of mouse external genitalia: implications for a role of estrogen in sexual dimorphism of the mouse genital tubercle. J Urol 2010;184(4 Suppl):1604-9.

11. Lee PA, Nordenström A, Houk CP, Ahmed SF, Auchus R, Baratz A, et al. S; global DSD update consortium. Global disorders of sex development update since 2006: perceptions, approach and care. Horm Res Paediatr 2016;85:158-80.

12. Ahmed SF, Bashamboo A, Lucas-Herald A, McElreavey K. Understanding the genetic aetiology in patients with XY DSD. Br Med Bull 2013;106:67-89.

13. Ahmed SF, Achermann JC, Arlt W, Balen A, Conway G, Edwards Z, et al. Society for Endocrinology UK guidance on the initial evaluation of an infant or an adolescent with a suspected disorder of sex development. Clin Endocrinol 2016;84:771-88.

14. Baetens D, Mladenov W, Delle Chiaie B, Menten B, Desloovere A, lotova $\mathrm{V}$, et al. Extensive clinical, hormonal and genetic screening in a large consecutive series of $46, \mathrm{XY}$ neonates and infants with atypical sexual development. Orphanet J Rare Dis 2014;9:209.

15. Bangalore Krishna K, Houk CP, Lee PA. Pragmatic approach to intersex, including genital ambiguity, in the newborn. Semin Perinatol 2017;41:244-51.

16. León NY, Reyes AP, Harley VR. A clinical algorithm to diagnose differences of sex development. Lancet Diabetes Endocrinol 2019;7:560-74.

17. Kyriakou A, Lucas-Herald AK, McGowan R, Tobias ES, Ahmed SF. Disorders of sex development: advances in genetic diagnosis and challenges in management. Adv Genom Genet 2015;5:165-77.

18. Yatsenko SA, Witchel SF. Genetic approach to ambiguous genitalia and disorders of sex development: what clinicians need to know. Semin Perinatol 2017;41:232-43.

19. Nixon R, Cerqueira V, Kyriakou A, Lucas-Herald A, McNeilly J, McMillan $M$, et al. Prevalence of endocrine and genetic abnormalities in boys evaluated systematically for a disorder of sex development. Hum Reprod 2017;32:2130-7.

20. Kolesinska Z, Acierno J Jr., Ahmed SF, Xu C, Kapczuk K, SkorczykWerner $A$, et al. Integrating clinical and genetic approaches in the diagnosis of 46,XY disorders of sex development. Endocr Connect 2018;7:1480-90.

21. Audi L, Ahmed SF, Krone N, Cools M, McElreavey K, Holterhus PM, et al. Genetics IN endocrinology: approaches to molecular genetic diagnosis in the management of differences/disorders of sex development (DSD): position paper of EU COST Action BM 1303 'DSDnet'. Eur J Endocrinol 2018;179:R197-206. dslkw.

22. Yarrow JF, Beck DT, Conover CF, Beggs LA, Goldberger BA, Borst SE. Invalidation of a commercially available human $5 \alpha$-dihydrotestosterone immunoassay. Steroids 2013;78: 1220-5.

23. Wudy SA, Hartmann M, Solleder C, Homoki J. Determination of 17alpha-hydroxypregnenolone in human plasma by routine isotope dilution mass spectrometry using benchtop gas chromatography-mass selective detection. Steroids 2001;66: 759-62.

24. Kulle A, Krone N, Holterhus PM, Schuler G, Greaves RF, Juul A, et al. Steroid hormone analysis in diagnosis and treatment of DSD: position paper of EU COST Action BM 1303 'DSDnet'. Eur J Endocrinol 2017;176:1-9.
25. Hannema SE, de Rijke YB. Improving laboratory assessment in disorders of sex development through a multidisciplinary network. Sex Dev 2018;12:135-9.

26. Honour JW, Conway E, Hodkinson R, Lam F. The evolution of methods for urinary steroid metabolomics in clinical investigations particularly in childhood. J Steroid Biochem Mol Biol 2018;181:28-51.

27. Sikaris K, McLachlan RI, Kazlauskas R, de Kretser D, Holden CA, Handelsman DJ. Reproductive hormone reference intervals for healthy fertile young men: evaluation of automated platform assays. J Clin Endocrinol Metab 2005;90:5928-36.

28. Gassner D, Jung R. First fully automated immunoassay for antiMüllerian hormone. Clin Chem Lab Med 2014;52:1143-52.

29. Johannsen TH, Main KM, Ljubicic ML, Jensen T, Andersen HR, Andersen MS, et al. Sex differences in reproductive hormones during mini-puberty in infants with normal and disordered sex development. J Clin Endocrinol Metab 2018;103:3028-37.

30. Johannsen TH, Andersson AM, Ahmed SF, de Rijke YB, Greaves RF, Hartmann MF, et al. Peptide hormone analysis in diagnosis and treatment of differences of sex development: joint position paper of EU COST action 'DSDnet' and European reference network on rare endocrine conditions. Eur J Endocrinol 2020;182:1-15.

31. Auchus RJ, Chang AY. 46,XX DSD: the masculinised female. Best Pract Res Clin Endocrinol Metabol 2010;24:219-42.

32. Nimkarn S, Gangishetti PK, Yau M, New MI. 21-HydroxylaseDeficient congenital adrenal hyperplasia. In: Adam MP, Ardinger HH, Pagon RA, Wallace SE, Bean LJH, Stephens K, et al., editors GeneReviews $®$ [Internet]. Seattle (WA): University of Washington, Seattle; 2002 [updated 2016 Feb 4]. 1993-2020. PMID: 20301350.

33. Bertelloni S, Russo G, Baroncelli GI. Human chorionic gonadotropin test: old uncertainties, new perspectives, and value in $46, \mathrm{XY}$ disorders of sex development. Sex Dev 2018;12: 41-9.

34. Vestergaard ET, Schjørring ME, Kamperis K, Petersen KK, Rittig S, Juul A, et al. The follicle-stimulating hormone (FSH) and luteinizing hormone $(\mathrm{LH})$ response to a gonadotropin-releasing hormone analogue test in healthy prepubertal girls aged 10 months to 6 years. Eur J Endocrinol 2017;176:747-53.

35. Carretto F, Salinas-Vert I, Granada-Yvern ML, Murillo-Vallés M, Gómez-Gómez C, Puig-Domingo M, et al. The usefulness of the leuprolide stimulation test as a diagnostic method of idiopathic central precocious puberty in girls. Horm Metab Res 2014;46: 959-63.

36. Bangalore Krishna K, Fuqua JS, Rogol AD, Klein KO, Popovic J, Houk CP, et al. Use of gonadotropin-releasing hormone analogs in children: update by an international consortium. Horm Res Paediatr 2019;91:357-72.

37. Ledig S, Hiort O, Scherer G, Hoffmann M, Wolff G, Morlot S, et al. Array-CGH analysis in patients with syndromic and nonsyndromic $\mathrm{XY}$ gonadal dysgenesis: evaluation of array CGH as diagnostic tool and search for new candidate loci. Hum Reprod 2010;25:2637-46.

38. White S, Ohnesorg T, Notini A, Roeszler K, Hewitt J, Daggag H, et al. Copy number variation in patients with disorders of sex development due to 46,XY gonadal dysgenesis. PloS One 2011;6: e17793. Published 2011 Mar 7.

39. Kon M, Fukami M. Submicroscopic copy-number variations associated with $46, \mathrm{XY}$ disorders of sex development. Mol Cell Pediatr 2015;2:7. 
40. Croft B, Ohnesorg T, Sinclair AH. The role of copy number variants in disorders of sex development. Sex Dev 2018;12:19-29.

41. Bashamboo A, McElreavey K. Human sex-determination and disorders of sex-development (DSD). Semin Cell Dev Biol 2015; 45:77-83.

42. Baetens D, Mendonça BB, Verdin H, Cools M, De Baere E. Noncoding variation in disorders of sex development. Clin Genet 2017;91:163-72.

43. Camats N, Flück CE, Audí L. Oligogenic origin of differences of sex development in humans. Int J Mol Sci 2020;21:1809.

44. Baxter RM, Arboleda VA, Lee H, Barseghyan H, Adam MP, Fechner $\mathrm{PY}$, et al. Exome sequencing for the diagnosis of 46,XY disorders of sex development. J Clin Endocrinol Metab 2015;100:E333-44.

45. Dong Y, Yi Y, Yao H, Yang Z, Hu H, Liu J, et al. Targeted nextgeneration sequencing identification of mutations in patients with disorders of sex development. BMC Med Genet 2016;17:23. Published 2016 Mar 15.

46. Alhomaidah D, McGowan R, Ahmed SF. The current state of diagnostic genetics for conditions affecting sex development. Clin Genet 2017;91:157-62.

47. Wang H, Zhang L, Wang N, Zhu H, Han B, Sun F, et al. Nextgeneration sequencing reveals genetic landscape in $46, X Y$ disorders of sexual development patients with variable phenotypes. Hum Genet 2018;137:265-77.

48. Hughes LA, McKay-Bounford K, Webb EA, Dasani P, Clokie S, Chandran $\mathrm{H}$, et al. Next generation sequencing (NGS) to improve the diagnosis and management of patients with disorders of sex development (DSD). Endocr Connect 2019;8:100-10.

49. Jacobson JD, Willig LK, Gatti J, Strickland J, Egan A, Saunders C, et al. High molecular diagnosis rate in undermasculinized males with differences in sex development using a stepwise approach. Endocrinology 2020;161:bqz015.

50. Speiser PW, Arlt W, Auchus RJ, Baskin LS, Conway GS, Merke DP, et al. Congenital adrenal hyperplasia due to steroid 21-hydroxylase deficiency: an endocrine society clinical practice guideline. J Clin Endocrinol Metab 2018;103:4043-88.

51. Honour JW. 17-Hydroxyprogesterone in children, adolescents and adults. Ann Clin Biochem 2014;51:424-40.

52. Van der Kamp H, Oudshoorn C, Elvers B, van Baarle M, Otten B, Wit J, et al. Cuttoff levels of 17-alfa hydroxyprogesterone in neonatal screening for congenital adrenal hyperplasia should be based on gestational age rather than on birth weight. J Clin Endocrinol Metab 2005;90:3904-7.

53. Miller WL. Congenital adrenal hyperplasia: time to replace $170 \mathrm{HP}$ with 21-deoxycortisol. Horm Res Paediatr 2019;91:416-20.

54. Kamrath C, Hochberg Z, Hartmann MF, Remer T, Wudy SA. Increased activation of the alternative 'backdoor' pathway in patients with 21-hydroxylase deficiency: evidence from urinary steroid hormone analysis. J Clin Endocrinol Metab 2012;97: E367-75.
55. Neocleous V, Fanis P, Phylactou LA, Skordis N. Genotype is associated to the degree of virilization in patients with classic congenital adrenal hyperplasia. Front Endocrinol 2018;9:733. Published 2018 Dec 3.

56. Parsa AA, New MI. Steroid 21-hydroxylase deficiency in congenital adrenal hyperplasia. J Steroid Biochem Mol Biol 2017; 165:2-11.

57. Baronio F, Ortolano R, Menabò S, Cassio A, Baldazzi L, Di Natale V, et al. 46,XX DSD due to androgen excess in monogenic disorders of steroidogenesis: genetic, biochemical, and clinical features. Int J Mol Sci 2019;20:4605.

58. Lutfallah C, Wang W, Mason JL, Chang YT, Haider A, Rich B, et al. Newly proposed hormonal criteria via genotypic proof for type II 3beta-hydroxysteroid dehydrogenase deficiency. J Clin Endocrinol Metab 2002;87:2611-22.

59. Khattab A, Haider S, Kumar A, Dhawan S, Alam D, Romero R, et al. Clinical, genetic, and structural basis of congenital adrenal hyperplasia due to 11ß-hydroxylase deficiency. Proc Natl Acad Sci U S A 2017;114:E1933-E1940.

60. Parajes S, Loidi L, Reisch N, Dhir V, Rose IT, Hampel R, et al. Functional consequences of seven novel mutations in the CYP11B1 gene: four mutations associated with nonclassic and three mutations causing classic 11 beta\}-hydroxylase deficiency. J Clin Endocrinol Metab 2010;95:779-88.

61. Mendonca BB, Leite MV, de Castro M, Kino T, Elias LL, Bachega TA, et al. Female pseudohermaphroditism caused by a novel homozygous missense mutation of the GR gene. J Clin Endocrinol Metab 2002;87:1805-9.

62. Quaynor SD, Stradtman EW Jr., Kim HG, Shen Y, Chorich LP, Schreihofer DA, et al. Delayed puberty and estrogen resistance in a woman with estrogen receptor $\alpha$ variant. N Engl J Med 2013;369: 164-71.

63. Burkhard FZ, Parween S, Udhane SS, Flück CE, Pandey AV. P450 Oxidoreductase deficiency: analysis of mutations and polymorphisms. J Steroid Biochem Mol Biol 2017;165:38-50.

64. Reisch N, Taylor AE, Nogueira EF, Asby DJ, Dhir V, Berry A, et al. Alternative pathway androgen biosynthesis and human fetal female virilization. Proc Natl Acad Sci U S A 2019;116:22294-9.

65. Parween S, Fernández-Cancio M, Benito-Sanz S, Camats N, Rojas Velazquez MN, López-Siguero JP, et al. Molecular basis of CYP19A1 deficiency in a 46,XX patient with R550W mutation in POR: expanding the PORD phenotype. J Clin Endocrinol Metab 2020;105:dgaa076.

66. Lin L, Ercan O, Raza J, Burren CP, Creighton SM, Auchus RJ, et al. Variable phenotypes associated with aromatase (CYP19) insufficiency in humans. J Clin Endocrinol Metab 2007;92:982-90.

Nota del artículo: La versión traducida del artículo puede encontrarse aquí: https://doi.org/10.1515/almed-2021-0042. 